BOEHLER, Carolina; MONTIEL, Juan Pablo: “¿Cómo testear la adecuación de un programa de compliance? Introducción al "modelo de los tres filtros"”.

Polít. Crim. Vol. 16, No 31 (Junio 2021), Art. 8, pp. 197-219

[http://politcrim.com/wp-content/uploads/2021/05/Vol16N31A8.pdf]

\title{
¿Cómo testear la adecuación de un programa de compliance? Introducción al "modelo de los tres filtros"
}

\section{How to Test the Adequacy of Compliance Programmes? An Introduction to the "Three Filters Model"}

\author{
Carolina Boehler \\ Abogada (Universidad de Buenos Aires) \\ Especialista en compliance y consultor \\ carolina.boehler@gmail.com \\ Juan Pablo Montiel \\ Doctor Europeo en Derecho Penal (Universidad Pompeu Fabra) \\ Director de CRIMINT \\ jpmontiel@crimint.org \\ https://orcid.org/0000-0003-0539-171X
}

\section{Resumen}

Fecha de recepción: 18/05/2020.

Fecha de aceptación: 28/08/2020.

La mayoría de las regulaciones adoptadas a nivel internacional considera que únicamente los programas de compliance que resultan adecuados son capaces de incidir favorablemente en la determinación de la pena para personas jurídicas. Sin embargo, existe todavía una gran incertidumbre respecto a los criterios que resultan decisivos a la hora de identificar esa adecuación. La contribución vincula la problemática con varias cuestiones de actualidad (por ejemplo, el valor de las certificaciones de programas de compliance) y desarrolla un esquema de razonamiento de tres pasos, con el que se clarifican las condiciones que deben satisfacerse para la existencia de un modelo de prevención adecuado.

Palabras claves: normas ISO, sistema de detección, programas de compliance, corrupción, behavioral compliance.

\begin{abstract}
Most regulations adopted at the international level consider that only adequate compliance programs can have a favorable impact on the determination of the sanction for legal entities. However, there is still a great deal of uncertainty regarding the criteria to determine such adequacy. The article links this problem with several current issues (for example, the value of compliance program certifications) and develops a three-step reasoning scheme, clarifying the conditions that must be satisfied for the existence of an adequate prevention model.
\end{abstract}

Keywords: Adequacy, ISO standards, internal investigations, detection system, compliance programs, corruption, behavioral compliance 


\section{Introducción: del "make-up-compliance" al "compliance real"}

Los fenómenos del compliance y de la responsabilidad administrativa o penal de las personas jurídicas aparecen indisolublemente ligados, tanto desde el punto de vista conceptual como también práctico. Sea para fundamentar la responsabilidad de la persona jurídica o bien para excluirla, los programas de compliance desempeñan un rol decisivo. ${ }^{1}$

Desde un punto de vista conceptual, la vinculación trasciende el modelo de atribución escogido. ${ }^{2}$ Para los defensores del modelo de la autorresponsabilidad la falta de implementación de un programa de compliance o su ineficaz implementación es una circunstancia constitutiva del defecto organizativo que constituye el objeto del injusto de la responsabilidad de la persona jurídica. ${ }^{3}$ Por su parte, la falta de compliance representa uno de los criterios decisivos de atribución de responsabilidad en los modelos de la heterorresponsabilidad. ${ }^{4}$ Pero también la praxis y las estrategias de política criminal afirman esta conexión entre compliance y la responsabilidad de la persona jurídica: el enforcement de los programas de cumplimiento se evaporaría si no pudiese sancionarse a la persona jurídica, ${ }^{5}$ del mismo modo que una adecuada implementación de un sistema sancionatorio público requiere de la colaboración de los entes colectivos mediante herramientas de autorregulación.

Nuestra contribución se concentra en los efectos que tiene el compliance en la eliminación de la responsabilidad (administrativa o penal) de la persona jurídica, especialmente en la identificación de aquellos criterios que permiten determinar la adecuación de estos sistemas para dar lugar a una exención de pena. Es importante advertir aquí que a lo largo de los años la relación entre compliance y responsabilidad sancionatoria de las personas jurídicas fue variando significativamente de cara a la exclusión de la sanción, arrancando con la exigencia de la mera presencia de algunos elementos de compliance, pasando luego por la exigencia de un sistema de compliance, hasta finalizar en la demanda de un sistema de compliance adecuado. ${ }^{6}$ Como es bien sabido por todos, en la actualidad prima a nivel comparado la tesis de que la impunidad de las personas jurídicas únicamente puede tener lugar cuando el sistema de compliance es eficaz para obstaculizar seriamente la comisión de delitos. En caso de no gozar de esta característica, por tratarse de un "compliance cosmético", 7 la organización deberá resignarse en el mejor de los casos a verse favorecida con una atenuación de la sanción. Ahora bien, pese a ser abiertamente dominante este criterio a nivel global, todavía existen grandes lagunas relativas a los parámetros que una empresa, un fiscal o un juez deben seguir para saber si un programa de compliance concreto es o no adecuado. Incluso cuando a nivel comparado se pueden identificar

\footnotetext{
${ }^{1}$ Ciertamente el non-compliance configura el injusto de la persona jurídica o bien es el motivo de imputación del hecho de la persona física a la jurídica. Con todo, el compliance per se puede operar como una eximente. KUBICIEL (2015), pp. 69 y ss. Así también en España con la Circular de la Fiscalía General del Estado $\mathrm{N}^{\circ} 1 / 2016$, pp. 64 y ss.

${ }^{2}$ Esclarecedor resulta el análisis de HILF (2015), pp. 63 y ss., quien muestra que, más allá de los diferentes modelos de imputación (en su caso, analiza los seguidos en Austria, Lichtenstein y Suiza), el compliance desempeña un rol importante para determinar el defecto de organización (Organisationsverschulden).

${ }^{3}$ GÓMEZ-JARA DIÉZ (2016), p. 40; NIETO MARTÍN (2013), p. 37.

${ }^{4}$ Sobre la incidencia de los programas de compliance en los modelos de hétero-responsabilidad, PALMA HERRERA (2014), pp. 157 y ss.

${ }^{5}$ MONTIEL (2018b), pp. 180 y ss.

${ }^{6}$ CHEN y SOLTES (2018), passim. .

${ }^{7}$ HEMPHILL y CULLARI (2009), p. 345.
} 


\section{Polít. Crim. Vol. 16, № 31 (Junio 2021), Art. 8, pp. 195-216 \\ [http://politcrim.com/wp-content/uploads/2021/05/Vol16N31A8.pdf]}

numerosos documentos que precisan algunos criterios, todavía persisten importantes niveles de incertidumbre.

El presente trabajo enfoca este problema de manera global y desde esta perspectiva analiza los diferentes sistemas seguidos a nivel comparado para determinar la adecuación de un programa de compliance. Sobre la base de este análisis y de la crítica a estos sistemas, se desarrolla una propuesta que parte de un esquema de razonamiento que damos en llamar "modelo de los tres filtros". Si bien se parte de un cuidadoso análisis conceptual, el artículo está pensando para académicos y prácticos, en el afán de dotar a estos últimos de elementos concretos que le permitan saber qué tan potente resulta un determinado programa de compliance para conllevar una exoneración de responsabilidad de la persona jurídica.

\section{Punto de partida para el análisis de la adecuación: método tradicional y abreviado}

Una mirada atenta de algunos de los regímenes legales de responsabilidad penal/administrativa de persona jurídica en Latinoamérica, América del Norte y Europa muestra algunos esfuerzos de organismos públicos para precisar aquellos elementos o criterios que deben verse satisfechos para concluir que un programa de compliance es adecuado para excluir la pena. En algunos casos ello se expresa en leyes o en lineamientos (Guidelines) de entidades públicas que establecen criterios pormenorizados para medir la eficacia del programa de integridad. Frente a todo ello aparece recientemente la pregunta acerca del papel que desempeñan las certificaciones que en la actualidad están proliferando en diferentes regiones. ${ }^{8}$ Nos referimos concretamente a la posibilidad de certificar bajo normativa técnica ya sea nacional o internacional (a modo de ejemplo: ISO, ${ }^{9}$ y UNE en el caso español, respectivamente.) la calidad de programas de compliance en general y anticorrupción y tributario, en particular. Sin dudas la pregunta que surge aquí es si la sola demostración de una certificación resulta capaz de bloquear cualquier intento de responsabilizar a una persona jurídica. ${ }^{10}$

En primer lugar, es importante mostrar que en general se advierte en diferentes países un método de identificación de la adecuación que podría caracterizarse como tradicional. Este método tradicional se caracterizaría por exigir de parte del aplicador del derecho una labor más o menos ardua, consistente en estudiar si el sistema de compliance en cuestión resulta adecuado. Aquí el juez o fiscal deberá analizar caso por caso si el modelo de prevención satisface determinados estándares y recién a partir de ello determina su eficacia o ineficacia. Como veremos más adelante, los jueces o fiscales deben atenerse a una guía específicamente prevista a tal efecto o bien tomar en consideración simplemente criterios generales, según la legislación respectiva. Lo que caracteriza a este método es que aquella persona encargada de juzgar la adecuación del compliance está obligada a estudiar el sistema concreto que tiene una persona jurídica y a partir de allí determinar si efectivamente era capaz de obstaculizar seriamente la comisión de delitos. Para llegar a

\footnotetext{
${ }^{8}$ Sobre esta cuestión en diferentes países europeos (y especialmente en Alemania), SCHEFOLD (2017), pp. 27 y ss.

9 Vale recordar que entre las normas ISO que contienen recomendaciones en relación a corrupción, compliance y cohecho pueden citarse ISO 26000:2010, ISO 19600:2014 y naturalmente la ISO 37001:2016. Al respecto, confróntese BERNDT y JABLOWSKI (2019), pp. 112 y ss.

${ }^{10}$ Sobre éstas y otras cuestiones vinculadas a las certificaciones, confróntese CHEREPANOVA (2019), passim.
} 
esta conclusión se vale de diferentes criterios, pero en cualquier caso se requiere un involucramiento activo del juez o fiscal en el análisis pormenorizado de algunos elementos del programa de compliance o, al menos, de algunos específicos.

Por contraposición, la aparición de las certificaciones impulsa la consideración de un segundo método que podríamos denominar abreviado. En las versiones más extremas de este método abreviado la certificación sería un indicador absolutamente fiable de que el programa en cuestión satisface las condiciones necesarias para ser tenido como adecuado. Desde esta perspectiva, quien desea acreditar la adecuación de un sistema de compliance para sobreseer o absolver a una persona jurídica debería remitirse simplemente a la constancia de certificación. No sería necesario entonces hacer un estudio pormenorizado del concreto sistema, sino que la entidad certificadora habría ya realizado ese trabajo y habría constatado la eficacia. Según la versión extrema del fenómeno de las certificaciones, cabría afirmar que todo sistema de compliance certificado (por ejemplo) bajo normas ISO gozaría de una presunción iure et de iure de adecuación. Más allá que a nivel global innumerables empresas ofrecen los servicios de certificación, cabe destacar que específicamente en Chile la Ley $\mathrm{N}^{\circ} 20.393$ prevé la posibilidad de que las empresas obtengan una certificación de sus sistemas de compliance (art. 4, 4.). ${ }^{11}$ Más allá de esta previsión legal, no está claro cuál es el papel que estas certificaciones puedan desempeñar en un proceso penal contra la persona jurídica.

Con todo, cada vez con mayor frecuencia es posible encontrar voces en la doctrina que cuestionan el valor de las certificaciones y que, en definitiva, estiman inconveniente hablar de lo que se caracteriza aquí como "método abreviado". ${ }^{12}$ Por empezar, la praxis judicial ya nos muestra casos en los que personas jurídicas empiezan a ser perseguidas penalmente, pese a contar con un modelo de prevención certificado con normas de calidad. ${ }^{13} \mathrm{Al}$ mismo tiempo, también en el ámbito regulatorio se aprecia cierto escepticismo respecto al valor de estas certificaciones. ${ }^{14}$ En nuestra opinión, ello fue lo que sucedió en Perú con las reglas establecidas en la Ley No 30.424 y su posterior Decreto Supremo No 002-10-JUS, donde se acabó reduciendo sensiblemente el valor asignado a las certificaciones. ${ }^{15}$ En realidad, conviene no olvidar que las certificaciones de calidad

\footnotetext{
${ }^{11}$ Sobre el sistema de certificación chileno, confróntese HERNÁNDEZ BASUALTO (2017), pp. 226 y ss.

${ }^{12}$ Confróntese entre otros GARCÍA CAVERO (2017), pp. 153 y ss.

${ }^{13}$ Los casos de Eni y Legg Masson son especialmente representativos en este sentido, pues a las pocas semanas de haber obtenido la certificación ISO 37001 encontraron a sus directivos procesados o suscribiendo NPA (non prosecution agreements) con el DOJ por violaciones a la FCPA.

${ }^{14}$ Una de las razones de este escepticismo tiene que ver con que, pese a que en las certificaciones se sigue el mismo proceso y metodología, ellas no son realizadas por las mismas entidades certificadoras, por lo que el resultado puede ser diverso. Adicionalmente, si se tiene en consideración que ISO habilita el acceso a un proceso abreviado de certificación en las normas de 37.001 cuando las compañías hubiesen obtenido con anterioridad la certificación correspondiente a alguno de los estándares 9001 Calidad, 14000 Medio Ambiente, y/ o 50001, los resultados pueden no ser del todo deseables. Al respecto, CHEREPANOVA (2019), passim.

${ }^{15}$ Concretamente en Perú el art. 19 de la Ley $N^{\circ} 30.424$ de responsabilidad administrativa de personas jurídicas establece que los modelos de integridad pueden estar certificados por terceros debidamente registrados y autorizados, pero en la Reglamentación los alcances reales de la certificación se ven notablemente reducidos. Así, el art. 49 del Decreto reglamentario No 002-2019-JUS fija que la autoridad encargada de emitir un informe técnico sobre la adecuación del programa de compliance es la Superintendencia del Mercado de Valores, institución que podrá considerar la existencia de certificaciones a la hora de elaborar su informe. De este modo, en Perú también el efecto de las certificaciones se ve limitado, primero, por el hecho de que en la consideración del juez vale más (implícitamente) el informe
} 
al estilo ISO o UNE simplemente acreditan estandarización de procedimientos y no necesariamente una mirada profunda sobre la calidad (en este caso) de un sistema de compliance antisoborno. ${ }^{16}$ En este punto resulta crucial entender que una certificación se realiza sobre la base de una "foto" que se toma durante un período determinado de tiempo, sin contemplar la dimensión más dinámica del proceso y de la vida empresarial. En definitiva, el denominado "método abreviado" únicamente parece tener sentido en el plano conceptual, pero en la praxis resulta ser inaceptable y en cualquier caso absolutamente desaconsejable. No obstante, ello no debería llevarnos a negar toda relevancia práctica a las certificaciones, sino que, en el mejor de los casos, a darle su justo valor. Como entienden Montaner y Fortuny al analizar las normas de calidad UNE 19601, estas certificaciones tienen una clara utilidad procesal el permitir acreditar la adopción y ejecución de un modelo de gestión con carácter general y además posibilitar a las defensas en determinadas circunstancias acreditar de manera tangible la existencia de una cultura de cumplimiento real en la organización. ${ }^{17}$ Pero, concretamente de cara a la exención de la responsabilidad (penal o administrativa) de la persona jurídica, una certificación a lo sumo puede servir como un mero indicio de diligencia en materia de prevención de riesgos. ${ }^{18}$

\section{Estrategias de identificación de la adecuación de un sistema de compliance. Modelos reconocibles a nivel comparado}

Recientemente el legislador argentino introdujo, en los artículos 22 y 23 de la Ley 27.401, una serie de criterios para determinar el carácter adecuado de un programa de compliance. Siguiendo la tendencia mundial, la adecuación de un programa de integridad — sumada a otros requerimientos, tales como la denuncia espontánea y la devolución del beneficio indebidamente obtenido (art. 9 Ley 27.401) - conlleva en el Derecho argentino la exención de la responsabilidad penal de la persona jurídica. Concretamente los preceptos mencionados especifican qué elementos debe tener un programa de compliance para ser juzgado como adecuado. Complementariamente, la Oficina Anticorrupción argentina publicó unos "Lineamientos de Integridad", bajo el formato de una guía práctica para las empresas (principalmente), abogados y funcionarios judiciales, mediante los cuales se busca precisar de qué modo implementar en la praxis los criterios suministrados por el legislador. Con ello, el Estado argentino ha procurado establecer algunas pautas que den certeza jurídica a los destinatarios de la norma, pese a los múltiples problemas que padece esta regulación.

La descripción de la situación legal argentina sirve como disparador del análisis de posibles sistemas de identificación de la adecuación del compliance reconocibles en los diferentes países que regulan la responsabilidad (penal/administrativa) de la persona jurídica. A grandes rasgos, creemos posible diferenciar dos grandes estrategias, las que podrían ser caracterizadas como sistema de valoración tabulada, por un lado, y sistema de valoración discrecional, por el otro. Si bien la tendencia hacia el sistema mencionado en primer orden se está convirtiendo claramente en mayoritaria —en nuestra opinión fruto de la elevación de los estándares de compliance a nivel global—, sirve tomar en

\footnotetext{
de la Superintendencia del Mercado de Valores que las certificaciones y, luego, por autorizar a esta institución a tenerlas en cuenta al momento del informe o directamente no hacerlo.

${ }^{16}$ SCHEFOLD (2017), pp. 27 y ss.; MONTANER y FORTUNY (2018), pp. 3-9.

${ }^{17}$ MONTANER y FORTUNY (2018), p. 14.

${ }^{18}$ En este sentido, GARCÍA CAVERO (2017), p. 154.
} 
consideración la posibilidad de un segundo sistema, para comprender mejor el abordaje de la temática que se sigue en otros países.

El denominado sistema de valoración tabulada se caracteriza por la existstencia de criterios ex ante objetivos y externos al aplicador del Derecho. Esto significa que la determinación de la adecuación del sistema de compliance no se da caso por caso atendiendo a las características particulares de cada situación, sino que existen parámetros previos de medición que condicionan la valoración del fiscal o juez. De este modo, el sistema prevé regulaciones que suministran al aplicador del Derecho criterios ex ante específicos para saber si una persona jurídica cuenta o no con un programa de prevención adecuado. Estos parámetros externos establecen las características o elementos que debe contener todo sistema de compliance para ser adecuado, con independencia de las notas particulares del concreto caso y de la concreta persona jurídica. Bajo este sistema el aplicador del Derecho ve simplificado su trabajo, pues no se ve forzada a realizar un análisis exhaustivo de toda la estructura de compliance de la persona jurídica, sino únicamente se debe mirar si se satisfacen los criterios específicos previstos en la regulación y, en caso de no verse satisfechos, se estaría en condiciones de concluir que existe un déficit de organización. Cabe remarcar que estos criterios específicos no deben estar plasmados exclusivamente en la ley, sino que también se observa que en algunas regulaciones administrativas - las denominadas Guidelines - también se da una clara tabulación sobre lo que debe buscar un juez o fiscal para concluir si el sistema de compliance en cuestión amerita la impunidad de la persona jurídica.

Bajo este sistema puede advertirse al mismo tiempo que conviven dos maneras distintas de tabular los criterios. Por un lado, tenemos una tabulación de elementos, en la que se determinan cuáles son los elementos mínimos que debe tener todo modelo de prevención para ser adecuado. Este es el caso de la ley argentina, donde la Ley $\mathrm{N}^{\circ} 27.401$ establece que para que un sistema de compliance antisoborno sea adecuado debe contar por lo menos con un código de ética o conducta, un protocolo para prevenir ilícitos en procesos de contratación con el Estado y capacitaciones periódicas sobre el programa de compliance (art. 23). ${ }^{19}$ Según el tenor literal de la ley, basta con que se constate la presencia de estos elementos para concluir que el programa es adecuado. ${ }^{20}$

Por otro lado, puede reconocerse una tabulación funcional que no fija los elementos mínimos que todo sistema de compliance debe exhibir para ser adecuado, sino que orienta al aplicador del Derecho para que examine si el sistema cumple ciertas funciones de manera adecuada. Este examen por funciones conlleva analizar si un concreto programa cumple satisfactoriamente con determinadas funciones, con independencia de si se posee una determinada cantidad de elementos. Ello puede llevar a que, dependiendo de las características de la organización, esas funciones sean cumplidas por un elemento o por varios al mismo tiempo. A nuestro entender, el prototipo de este tipo de tabulación lo encontramos en los Guidelines del Departamento de Justicia (DOJ) de los Estados Unidos

\footnotetext{
${ }^{19}$ En nuestra opinión también sigue este sistema el legislador peruano a partir del art. 17 Ley No 30.424.

${ }^{20}$ Con todo, dados los problemas derivados de hacer una incorrecta selección de los elementos mínimos, la Oficina Anticorrupción argentina intentó “suavizar" la noción de elementos mínimos para que en la praxis los estándares de adecuación fueran más exigentes. Confróntese, Lineamientos de Integridad para el mejor cumplimiento de lo establecido en los artículos 22 y 23 de la ley n ${ }^{\circ} 27.401$ de responsabilidad penal de las personas jurídicas, Sección 2.3. Más allá de este loable intento y de que compartimos la solución dada por la Oficina Anticorrupción, no debe pasar por alto que tal interpretación difícilmente pueda verse como secundum legem. Al respecto, en detalle MONTIEL (2018a), pp. 12 ss.
} 
de América. El documento describe los factores específicos que los fiscales deben considerar al llevar a cabo una investigación de una corporación. Teniendo en cuenta que la investigación tiene lugar una vez cometido el ilícito, la guía cumple la función de ayudar a los fiscales a determinar si el programa de compliance de la compañía — pese a la comisión del delito - era efectivo al momento de la ofensa o y si lo es al momento de la resolución de acusación. La finalidad es determinar de forma apropiada cualquier resolución o enjuiciamiento, sanción pecuniaria si correspondiere y obligaciones de cumplimiento contenidas en cualquier resolución penal de la corporación. ${ }^{21}$

Sin dudas, una de las grandes ventajas de este sistema tiene que ver con razones de economía procesal, puesto que un juez o fiscal no se ve forzado prima facie a estudiar exhaustivamente un programa de compliance para recién ahí formarse una idea sobre su adecuación para activar la impunidad de las persona jurídica, sino que basta con que se detenga a analizar si se satisfacen los mínimos identificados por la norma (los elementos o las funcionalidades). En caso de no estar presentes estos mínimos, será procedente formular formalmente la correspondiente acusación. A simple vista, ello contribuye también a ganar en certeza jurídica, respecto a la aplicación del Derecho y a lo que se espera de la auto-organización empresarial.

Más allá de esta importante ventaja no conviene pasar por alto los inconvenientes que plantea el sistema en los casos de una tabulación de elementos, dado que la presencia de ciertos elementos mínimos puede ser un mal indicio de la adecuación de un programa. Una primera limitación surge de atender a una pura formalidad (la presencia de un determinado elemento) sin preguntarse sobre cómo funciona realmente. Es fácilmente imaginable la situación de una organización que cuente con determinados elementos importantes de un sistema de compliance (verbigracia, canales de denuncia, oficial de cumplimiento o protocolos de vinculación con el sector público), que sin embargo funcionen defectuosamente. En este punto una tabulación funcional presenta claras ventajas. Los problemas de una tabulación de elementos se ven aún más agravados cuando se hace una incorrecta selección de los elementos mínimos. Claramente esta situación es la que se presenta en Argentina, donde el legislador hizo una pésima selección de los elementos mínimos de un sistema de compliance. ${ }^{22}$ La exigencia de contar con un código de ética o conducta, un protocolo para prevenir ilícitos en procesos de contratación con el Estado y capacitaciones periódicas sobre el programa de compliance puede ser razonable para personas jurídicas de pequeñas o mínimas dimensiones, pero en el caso de empresas más complejas se trata de una estándar de integridad excesivamente bajo. ${ }^{23}$ En este último caso, de ningún modo un programa que cumple con estas exigencias de la ley puede ser considerado eficaz para prevenir la corrupción.

Por su parte, frente al sistema de la valoración tabulada encontramos el de la valoración discrecional. En estos casos los aplicadores del Derecho no cuentan previamente con parámetros objetivos y externos que nos indiquen qué características puntuales deben constatarse para concluir que un sistema de compliance es adecuado. Más bien, fiscales $\mathrm{y}$ jueces deben atender al caso concreto y analizar qué tan eficazmente funcionaba el

\footnotetext{
${ }^{21}$ FCPA (2018), passim.

${ }^{22}$ Críticos frente a este sistema en Argentina, MONTIEL (2018c), pp. 75 y ss.; AGUSTINA y VARGAS OVALLE (2019), pp. 87 y ss.; CORIA CARO y SOTA SÁNCHEZ (2019), p. 105.

${ }^{23}$ Por el contrario, parecen mucho más elevados los mínimos establecidos en Perú en el art. 17. 2 Ley № 30.424 .
} 
modelo de prevención. En estos casos no basta con analizar la presencia de ciertos elementos, sino que debe estudiarse en su conjunto el funcionamiento del sistema y luego concluirse su adecuación o no sobre la base del criterio del juez. ${ }^{24}$ Este sistema había sido previsto inicialmente en el Anteproyecto de la Ley $\mathrm{N}^{\circ} 27.401$, presentado por el gobierno argentino, que luego fue modificado drásticamente en el parlamento. Según entendemos, se trata del sistema seguido de manera dominante a nivel comparado y así lo encontramos, por ejemplo, en España y Chile. ${ }^{25}$

Claramente bajo este sistema la determinación de la adecuación se torna mucho más laboriosa que en el sistema anterior, pues el juez no cuenta con un "check-list" que deba seguir para llegar a una decisión, sino que debe analizar minuciosamente la funcionalidad del sistema en su conjunto. Al mismo tiempo, la falta de criterios objetivos y externos, que condicionen ex ante la decisión del fiscal o juez, disminuye claramente la certeza jurídica. En este sentido, una tabulación funcional se muestra como la opción más viable, porque además de mirar al sistema en su funcionamiento real, ofrece parámetros externos de decisión mucho más fiables. Sin embargo, no nos caben dudas de que, pese a los defectos que puede tener un sistema de valoración discrecional, este resulta mucho más aconsejable que el de tabulación por elementos, ya que en este último caso aumentan exponencialmente los "falsos positivos", es decir, casos de empresas claramente deficitarias en estructuras de prevención de ilícitos pero que "están bien de papeles".

\section{Modelo de los tres filtros}

\subsection{Bases del modelo}

Lo que denominamos aquí modelo de los tres filtros no es otra cosa que un esquema de razonamiento que se propone para poder medir si un sistema es adecuado o no para obstaculizar seriamente la comisión de delitos. Se trata de un conjunto de pasos que un fiscal o un juez debería seguir en su razonamiento para determinar si corresponde acusar o condenar a una persona jurídica (penal o administrativamente) o bien los pasos que debe seguir en su razonamiento un empresario para saber en qué lugar está parado de cara al compliance dentro de su organización. Como podrá apreciarse, el modelo combina diferentes elementos en la búsqueda de acercar una mirada holística y más global del problema y así traer herramientas conceptuales más sofisticadas a la práctica del compliance. $^{26}$

\footnotetext{
${ }^{24}$ Respecto a la práctica de los tribunales y fiscalías de los EEUU y otros países europeos en relación a la identificación de los criterios de adecuación de los programas de compliance, confróntese MUÑOZ DE MORALES ROMERO (2013), pp. 216 y ss.

${ }^{25}$ Vale recordar que en España la Circular 1/2016 de la Fiscalía General del Estado, que establece criterios de aplicación de la responsabilidad penal de las personas jurídicas, al igual que en Chile la Unidad Especializada Anticorrupción del Ministerio Público ha elaborado un importante documento titulado "Guía Práctica. Buenas Prácticas de Investigación. Responsabilidad penal de las personas jurídicas”. Si bien ambos documentos podrían hacernos pensar que en estos países se sigue un sistema más próximo a la valoración tabulada, a partir de una tabulación funcional, consideramos que esta apreciación sería incorrecta. Entendemos que en realidad son documentos que ofrecen criterios muy generales destinados a los fiscales, para que pueden llevar adelante satisfactoriamente la investigación contra la persona jurídica. Ello no significa que ofrezcan parámetros precisos para evaluar la adecuación de los programas de compliance. Por ejemplo, el documento de la Unidad Especializada Anticorrupción de Chile tiene un apartado sobre la investigación de un modelo de prevención de delitos (pp. 19 y ss.), donde advierte de la importancia de que los programas de compliance sean eficaces. Sin perjuicio de ello, no presenta criterios de adecuación que condicionen ex ante la valoración de los fiscales.

${ }^{26}$ Una mirada similar se aprecia en el atractivo modelo favorecido por ZENTES (2017), pp. 165 y ss.
} 
El esquema de razonamiento del modelo de los tres filtros consta de tres pasos: un primer paso (filtro) en el que se analizan características generales de los programas, un segundo paso en el que se escrutan los elementos específicos y su funcionalidad y, finalmente, un tercer paso, en cuyo marco se toman en consideración los comportamientos de los integrantes de la organización en el marco del denominado behavioral compliance. La base del modelo presupone un seguimiento ordenado de estos pasos, lo que no implica que necesariamente deban ser seguidos en todo caso, pues cuando un programa de compliance no pasa el primer filtro no tiene sentido indagar en las siguientes etapas, lo mismo que sucede cuando el programa pasa el primer filtro, pero no así el segundo y, por ende, no se requiere un análisis en el tercer nivel. En este caso, cabe concluir que el modelo de prevención no es adecuado para verse beneficiado por la exoneración de responsabilidad, sin necesidad de acudir a los restantes filtros.

\subsection{Primer filtro: criterios generales}

Podría decirse que existe una suerte de máxima que establece como punto de partida de la evaluación la necesidad de que todo programa de compliance se ajuste a los riesgos propios de la actividad de la persona jurídica, su dimensión y su capacidad económica. Con esta primera exigencia general se expresa la necesidad de que los programas de cumplimiento sean diseñados e implementados con un anclaje en la realidad de la concreta persona jurídica y, por ello, un modelo de prevención nacería defectuoso cuando desde un inicio no se asienta en la estructura y la actividad de la específica empresa bajo análisis. ${ }^{27} \mathrm{Al}$ mismo tiempo es importante remarcar que una exigencia general de este tipo puede ser advertida en diferentes países tales como Argentina, ${ }^{28} \mathrm{Chile},{ }^{29}$ España, ${ }^{30}$ Estados Unidos, ${ }^{31}$ etc.

En primer lugar, la vinculación a los riesgos propios de la actividad debe manifestarse en dos niveles diferentes. Uno de ellos se refiere a la actividad comercial concreta de la persona jurídica o al tipo de actividad que desempeña y por ello, verbigracia, no es el mismo el sistema que requiere una empresa dedicada a la industria de la celulosa y el papel que un banco, o incluso el que requiere una fundación. Así, un excelente sistema de cumplimiento para una industria puede resultar completamente inadecuado para otra o incluso los protocolos de una sociedad comercial pueden resultar absolutamente inaplicables para personas jurídicas sin fines de lucro. ${ }^{32} \mathrm{El}$ segundo de los niveles de adecuación al riesgo de la concreta persona jurídica se refiere a que el programa de compliance forzosamente debe haberse proyectado sobre la base de un mapa de riesgos

\footnotetext{
${ }^{27}$ Por ejemplo, la modelación de la organización del área interna del compliance en una empresa va a deber estar sujeta también a estas variables, de allí que, por ejemplo, la dedicación horario de un oficial de cumplimiento (Compliance-Beauftragter), la cantidad de integrantes del equipo de compliance, deba ajustarse al tamaño, estructura y capacidad económica de la organización, confróntese KNIERIM (2015), nm. 16 y s.

${ }^{28}$ Art. 22, $2^{\mathrm{a}}$ parte Ley $\mathrm{N}^{\mathrm{o}}$ 27.401. Para un análisis de esta regulación en Argentina, confróntese entre otros MONTIEL (2018c): pp. 75 y ss.; MARTÍNEZ (2018) pp. 287 y ss.

${ }^{29}$ Guía Práctica. Buenas Prácticas de Investigación. Responsabilidad penal de las personas jurídicas, pp. 21, 23, especialmente al determinar las competencias del oficial de cumplimiento.

${ }^{30}$ Circular 1/2016 de la Fiscalía General del Estado de España, pp. 44, 47.

${ }^{31}$ U.S. Department of Justice, Evaluation of Corporate Compliance Programs, p. 10.

${ }^{32}$ Sobre las particularidades del compliance en el sector de las organizaciones sin fines de lucro, confróntese el todavía hoy vigente trabajo de DAWSON y DUNN (2006), pp. 36 y ss.
} 
elaborado para esa concreta empresa. ${ }^{33}$ Ese mapa de riesgo debe dar cuenta y haber comprendido y valorado los riesgos específicos de esa persona jurídica, y a partir de allí deberían estructurarse los restantes elementos del programa, sus sistemas de reportes, etc. ${ }^{34}$ Este es un dato muy importante no sólo para empresas que no tienen experiencia en compliance, sino también para filiales de empresas multinacionales que pretenden armar su modelo de prevención sin mapas de riesgos específicos e importando simplemente los protocolos y normativas de la casa matriz. Según este primer filtro en el análisis de la adecuación de los modelos de prevención, el programa de compliance que no se asienta sobre un mapa de riesgo (adecuadamente realizado) resulta absolutamente inadecuado. ${ }^{35}$

Igualmente, el tipo de procesos de vigilancia, detección y reacción empresarial frente a los delitos varía decisivamente según la dimensión de la entidad y ello explica que el nivel de complejidad nunca pueda ser igual en una PyME que en una multinacional con filiales en diferentes países. ${ }^{36}$ Existe así una primera manera de enfocar la cuestión de la dimensión que consiste en tomar como referencia la cantidad de empleados que trabajan en la concreta persona jurídica, lo que hace esperable que a mayor cantidad de empleados más complejo sea el programa de compliance. Con todo, la dimensión también podría enfocarse desde la envergadura de la exposición al riesgo, puesto que cabría imaginar una empresa con una escasa cantidad de empleados, pero con un volumen de riesgo significativo que ameritaría un modelo de prevención más sofisticado y complejo que para una PyME convencional.

La conexión entre la capacidad económica de la persona jurídica en cuestión y la calidad del sistema de compliance merece un comentario particular. No es infrecuente que se interprete a este requisito bajo el lema "menos compliance para empresas pobres", ${ }^{37}$ como si los riesgos de corrupción fuesen menores a los de las empresas de mayor capacidad económica. Toda organización debe gestionarse eficientemente también en materia de compliance, lo que implica que con el presupuesto disponible debe tener un programa adecuado a sus riesgos. El negocio debe serle rentable al empresario, contando entre los diferentes costos económicos de su actividad también el correspondiente al diseño y mantenimiento del programa de integridad. ¡El negocio debe serle rentable incluyendo el costo del compliance! En todo caso, lo que sí parece razonable es exigirle a aquellas empresas con capacidad económica hacer inversiones importantes, incluso yendo más allá de los mínimos de un sistema adecuado. ${ }^{38}$

\footnotetext{
${ }^{33}$ Tal es así que para ZENTES (2017), p. 165, el mapeo de riesgos constituye la base más elemental de todo sistema de compliance.

${ }^{34}$ ZENTES (2017), p. 166; GARCÍA CAVERO (2017), pp. 49 y ss.

${ }^{35}$ CORIA CARO y SOTA SÁNCHEZ (2019), p. 105.

${ }^{36}$ GÓMEZ-JARA DIÉZ (2010), p. 462, el tamaño y el nivel de complejidad organizativo tienen una notable incidencia en la propia identificación de la responsabilidad penal de las personas jurídicas, puesto que solamente a aquellas que detentan cierto grado de complejidad se les reconoce una esfera de autonomía que conlleva la imposición de la obligación de obrar con fidelidad al Derecho. Sobre esta cuestión, ver MONTIEL (2018d), pp. 133 y ss., con ulteriores referencias.

${ }^{37}$ En nuestra opinión, en esta dirección apunta en Argentina el documento de la Oficina Anticorrupción, Lineamientos de Integridad para el mejor cumplimiento de lo establecido en los artículos 22 y 23 de la ley $\mathrm{n}^{\circ} 27.401$ de responsabilidad penal de las personas jurídicas, Sección 2.2. III).

${ }^{38}$ MONTANER (2019), p. 95.
} 


\section{Polít. Crim. Vol. 16, № 31 (Junio 2021), Art. 8, pp. 195-216 \\ [http://politcrim.com/wp-content/uploads/2021/05/Vol16N31A8.pdf]}

\subsection{Segundo filtro: criterios específicos}

El segundo filtro o paso en el razonamiento de un fiscal o juez a la hora de analizar la adecuación de un programa de compliance consiste en enfocarse en sus componentes y en su funcionalidad. Un modelo de prevención adecuado a los riesgos de la empresa, a su dimensión y capacidad económica puede resultar, sin embargo, inadecuado si no dispone de todos los elementos requeridos o bien si estos no funcionan correctamente. Elementos de un sistema de compliance pueden ser, entre otros, las capacitaciones periódicas, un sistema de monitorio de riesgos, protocolos antisoborno, un código de ética, el apoyo de la alta gerencia (el denominado tone from the top), due dilligence de terceros y en procesos de transformación societaria, canales de denuncia, sistema de investigaciones internas y un oficial de cumplimiento. Aquí aparecen enunciados todos estos elementos a modo ejemplificativo, lo que significa que no todas las empresas están obligadas a tener un programa de integridad con todos estos elementos, sino que su presencia únicamente será requerida atendiendo a las características generales de la empresa.

Según nuestro parecer, existen elementos de los que se puede prescindir, puesto que otros pueden suplir su ausencia de manera igualmente eficaz. Un buen ejemplo de ello son los códigos de ética, cuya presencia tiene una importancia más bien de carácter simbólico o puramente comunicativo y su fuerza normativa es limitada. ${ }^{39}$ Estos constituyen documentos que expresan los más altos valores de la organización y a partir de allí inspiran y guían innumerables protocolos tendientes a resguardarlos. Sin embargo, como herramientas de prevención directa tienen per se una fuerza más bien limitada, lo que se aprecia más fuertemente cuando se los compara, por ejemplo, con protocolos de obsequios o know your customer. En nuestra opinión, gran parte del cuerpo normativo del compliance y especialmente los protocolos antisoborno, antilavado o antitrust suplen mucho más eficazmente cualquier código de ética. Lo que se pretende enfatizar aquí no es que estos códigos sean totalmente prescindibles, dado que como estrategia de concientización y comunicación tienen algún valor en la prevención. Simplemente, se trata de enfatizar que ante el caso infrecuente de una organización que no tenga un código de ética no se puede concluir que el sistema padece de un defecto grave que lo hace inadecuado, sin antes analizar otros elementos. De este modo, habría que rechazar tal conclusión en el caso de una organización que posee, por ejemplo, sólidos protocolos, canales de denuncia y un sistema de monitoreo de riesgos.

En este segundo filtro la pregunta crucial es a qué elemento debo prestar atención para medir la adecuación de un programa de compliance, lo que conlleva la pregunta no sólo por su presencia o ausencia, sino también por la calidad de funcionamiento. Desde una perspectiva que se ha vuelto clásica en la teoría del compliance, los elementos de un programa pueden clasificarse según su vinculación con la prevention, detection o la reaction. ${ }^{40}$ Es indiscutible que para juzgar la adecuación resulta imprescindible observar al programa como un todo sistemático, con diferentes partes que interaccionan entre sí y

\footnotetext{
${ }^{39}$ Así también, LEHMANN (2015), nm. 54. Ello sería el caso especialmente respecto a los códigos éticos que con mayor habitualidad se encuentran en las empresas, orientados a preceptos más generales. Señala MOLANDER (1987), p. 629, que en estos casos el enforcement se torna más dificultoso, a lo que agrega que ellos esperan más bien alcanzar sus objetivos mediante el empleo de genuinas medidas de enforcement complementarias. Sin embargo, STEVENS (2008), pp. 601 y ss., en mi opinión, sobre la base de caracterizar dentro de la noción de "códigos de ética" elementos que habitualmente son externos a ellos.

${ }^{40}$ Sobre la caracterización clásica de estas tres fases, confróntese REEB (2011), pp. 3 y ss.
} 
que conjuntamente trabajan para prevenir la comisión de ilicitudes. ${ }^{41}$ Así, por ejemplo, un juez o fiscal únicamente estará en condiciones de elaborar un juicio sobre la existencia o inexistencia de un defecto de organización cuando haya realizado una valoración global. No obstante, por razones de optimización de los tiempos y recursos resulta recomendable para fiscales o jueces atender en primer término a aquellos elementos que pertenecen a la dimensión de la detección de ilicitudes. ${ }^{42}$ Se trata de un consejo práctico que puede ahorrarle a jueces y fiscales esfuerzos y tiempos en el análisis de adecuación, puesto que un programa que carece de buenos elementos de detección o cuyos elementos tienen un funcionamiento defectuoso nunca puede ser adecuado.

Desde esta perspectiva, cobra una gran importancia, por ejemplo, prestar atención a los canales de denuncia. La configuración y funcionamiento del sistema de whistleblowing tiene mucho para decir respecto a la capacidad de detección de ilicitudes de una persona jurídica. ${ }^{43}$ En nuestra opinión, no resultaría razonable afirmar la adecuación si la organización cuenta con canales de denuncia propios pero no externalizados, cuando no se observa transparencia respecto al manejo de las denuncias o se establece fuertes desincentivos para denunciar delitos de la empresa. ${ }^{44}$

Algo similar sucede con el sistema de monitoreo de riesgo, donde un mal sistema de procesamiento de información puede hacer tremendamente vulnerable a la empresa de cara a evitación de hechos de corrupción. Por ejemplo, una empresa de importantes dimensiones que todavía realice un monitoreo "manual", sin un software complejo que arroje información de manera permanente o certera sobre los riesgos previstos en mapa de riesgo original o sobre una relocalización o nuevo riesgo, difícilmente pueda jactarse de contar con un sistema de detección potente. En esta dirección es importante que los investigadores y jueces presten también atención a los procesos de supervisión de terceros en el curso normal del negocio, pero también en las actividades de transformación empresarial, dado que en un proceso satisfactorio de due dilligence puede aparecer información relevante sobre la posible comisión de ilícitos. ${ }^{45}$ También aquí, la falta de procesos de due dilligence o un cumplimiento defectuoso ahorra a jueces y fiscales la tarea de indagar sobre otros aspectos del sistema de compliance a los efectos de determinar la adecuación.

Una mención especial merecen las investigaciones internas. Habitualmente estos mecanismos de pesquisas aparecen marginados a un lugar de importancia secundaria en un modelo de prevención, debido quizás a que su activación resulta algo contingente en

\footnotetext{
${ }^{41}$ NIETO MARTÍN (2015), pp. 80 y ss.

${ }^{42}$ En nuestra opinión, bajo la denominación de "test del debido control” también hace hincapié en el sistema de detección NIETO MARTÍN (2015), pp. 84 y ss., aunque consideramos que bajo la idea de control aparecen involucradas otras actividades distintas a la detección de ilicitudes, lo que le haría perder precisión al test. En nuestra opinión, la propuesta de Nieto Martín mantiene similitudes con los criterios de efectividad plasmados en las U.S. Sentencing Guidelines. Respecto a estos criterios, confróntese MOMSEN y TWEEN (2015), nm. 21, 23 y ss.

${ }^{43}$ GÓMEZ MARTÍN (2013), p. 130; ROTSCH (2015), nm. 28; BERMEJO (2013), p. 52.

${ }^{44}$ En este punto, no es casual que algunas de estas disfuncionalidades sean contraindicadas por numerosas Guidelines (a modo de ejemplo, US Department of Justice Criminal Division, Evaluation of Corporate Compliance Programs p. 5) o incluso impidan obtener la certificación en ISO 37.001 Anti-Bribery Management Systems, Nonconformity and Corrective Action, p. 66.

45 Justamente la U.S. Sentencing Guidelines establece como uno de los criterios determinantes para la efectividad del programa la existencia de componentes de due dilligence ( $\$ 8$ B 2. 1 [b]). Al respecto, confróntese MOMSEN y TWEEN (2015), pp. 1039 y s.
} 
la vida de una organización. Al mismo tiempo, suele catalogarse a las investigaciones internas dentro del espectro de la reaction, ${ }^{46}$ sobre la base de la particularidad con la que acaban al adoptarse sanciones o programas de indulgencias. Con todo, existen razones para revisar estas consideraciones preliminares. ${ }^{47}$

En primer lugar, desde el plano académico se ha buscado enfáticamente destacar el valor de las investigaciones internas en el risk management, ${ }^{48}$ al igual que en la praxis se ofrecen muestras claras sobre cómo se pueden potenciar las capacidades de un programa de compliance a partir de contar con un sistema idóneo de investigaciones internas. En nuestra opinión, una inadecuada implementación de internal investigations puede llevar a que un sistema de detección de ilicitudes pueda ser manifiestamente fallido, pese a contar con canales de denuncia adecuados a las mejores prácticas empresariales. Ello es así porque, por ejemplo, un mal sistema de investigaciones puede filtrar inadecuadamente aquellas denuncias merecedoras de investigación o bien tapar investigaciones relativas a denuncias que pesan sobre la alta gerencia. Estas fallas conllevan inevitablemente el fracaso de un sistema de compliance.

\subsection{Tercer filtro: behavioral compliance}

Un dato aparecido recientemente en los EE.UU. ha generado señales de alerta entre las compañías y los órganos públicos encargados de la persecusión penal de la corrupción: pese a que las empresas cada vez cuentan con estándares más altos en materia de compliance, el valor pagado en multas también ha ascendido significativamente. Según los registros del Department of Justice de los Estado Unidos (DOJ), ${ }^{49}$ en 2018 dieciséis compañías pagaron un récord de USD \$2,89 billones para resolver casos vinculados a la Foreign Corrupt Practices Act (FCPA). Esto incluye los montos prorrateados en resoluciones con el DOJ o la Security Exchange Commission (SEC) o ambos y a través de declinaciones del DOJ con acuerdos de restitución. En el 2017, otras once compañías pagaron por encima de USD \$1,92 billones tras verse involucradas en hechos de corrupción. Por su parte, en el año 2016 los montos pagados por veintisiete compañías involucradas en casos de corrupción alcanzaron la suma de USD \$2.48 billones. Lo más llamativo de esta información es que todas las empresas contaban con programas de compliance más o menos sólidos y que cumplían con las formalidades exigidas por el Estado. Más aún, muchas de ellas habían invertido fortunas en el diseño y la implementación de los programas. ${ }^{50}$

Son muy diversos los motivos exactos que llevaron a estas empresas a verse involucradas en estos hechos, pese a la existencia de programas prima facie sólidos. Ahora bien, la doctrina ha advertido que en la gran mayoría de los casos las organizaciones habían sido especialmente cuidadosas a la hora de dar cumplimiento a las exigencias puramente formales que imponía la autoridad de aplicación norteamericana, algo que no había

\footnotetext{
${ }^{46}$ Así lo entendí (erróneamente) hace algunos años, MONTIEL (2012), pp. 188 y s.

47 Especialmente en lo que se refiere a la inserción de las investigaciones internas en el sistema de compliance, sería preferible entender que se trata aquí de un mecanismo de detection. Así, REEB (2011), pp. 3 y ss. El segmento de la reaction aparece en realidad representado por medidas de tipo sancionatorio, las cuales justamente permiten darle a las investigaciones la aceptación (Akzeptanz) necesaria en la empresa, WAUSCHKUHN (2012), p. 68.

${ }^{48}$ MOMSEN (2015), nm. 1.

${ }^{49}$ FCPA (2018), passim.

${ }^{50}$ CHEN y SOLTES (2018), passim.
} 
sucedido a la hora generar un verdadero cambio cultural que incidiera en los valores o motivos que guían las conductas de sus miembros. ${ }^{51}$ En este sentido, se destaca que programas formalmente adecuados pueden, sin embargo, no asegurar una capacidad real de evitación real de ilícitos de la empresa. En 2013, por ejemplo, fue condenado Garth Peterson en el famoso caso que involucró a la firma Morgan Stanley por el pago de sobornos a funcionarios públicos chinos, quien contó además con la colaboración de otros empleados para poder burlar exitosamente los sistemas de controles internos. ${ }^{52} \mathrm{Si}$ bien la firma fue desligada de una responsabilidad penal por la fortaleza de su sistema de prevención y a partir de su compromiso de invertir en mejoras, el dato más llamativo fue que Garth Peterson había recibido siete entrenamientos de compliance, treinta y cinco acciones puntuales destinadas a evitar el pago de sobornos. ${ }^{53}$ En otras palabras, Morgan Stanley había sido un "buen alumno" frente a la DOJ, pese a lo cual no había logrado incidir verdaderamente en la conducta de sus empleados. Esta información invita a reflexionar entonces sobre si un programa de compliance completo y adecuado a las características de la organización resulta suficiente para prevenir eficazmente la corrupción. En todo caso, parece que un sistema con estas características resulta adecuado para salvarse de la multa, pero no así para prevenir delitos.

Este escenario muestra la necesidad de trascender un enfoque puramente formalista de la adecuación de los sistemas de compliance a otro que incorpore la dimensión de lo que más modernamente se denomina behavioral compliance, bajo la forma de un tercer filtro. ${ }^{54}$ Este cambio de enfoque obliga a dejar de entender a los modelos de prevención como una lista de tareas que debe ir tildándose en la medida en que se van cumpliendo los objetivos, sin interiorizar las razones de las tareas que se van realizando o sin tomar conciencia de los valores que subyacen a las prácticas exigidas. La introducción de la perspectiva del behavioral compliance invita a estudiar y trabajar sobre los motivos individuales que llevan a estafar u obrar de manera egoísta, incluso en circunstancias donde se sabe que se está realizando algo incorrecto y bajo el riesgo de ser descubierto.

¿Qué explica la existencia de programas de compliance altamente exigentes, que no obstante fallan a la hora de prevenir eficazmente? Todd Haugh ha advertido recientemente los problemas que trae aparejado el fenómeno de la "criminalización de los programas de compliance", esto es, la asunción por parte de las organizaciones de un enfoque de compliance presidido por los preceptos de la legislación penal, su modo de impartir justicia y su enforcement. ${ }^{55}$ En su opinión, al mimetizarse la autoregulación corporativa con el sistema penal mediante la imposición de sanciones cada vez más severas y un control excesivo se genera un efecto inverso al desatender el factor humano. ${ }^{56}$ Resulta totalmente contraproducente reducir el compliance a una sencilla tarea de tildar casilleros de políticas que ni siquiera invitan a reflexionar acerca de la propia

\footnotetext{
${ }^{51}$ PAINE (1994), passim.

${ }^{52}$ E.D. New York, United States of America v. Garth Peterson, de 28 de Agosto de 2013.

${ }^{53}$ CHEN y SOLTES (2018), passim.

${ }^{54}$ Incorpora también el enfoque del behavioral compliance en el análisis de la adecuación de los programas de integridad, KLEINHEMPEL (2018), pp. 219 y ss. Sin embargo, consideramos este autor parece querer reducir la fortalece del compliance a esta dimensión, algo que para nosotros resulta abiertamente inconveniente. La valoración de un programa de integridad depende de muchos factores más formales, distintos del behavioral compliance, como ser la presencia y funcionamiento de canales de denuncia, del sistema de investigaciones internas, etc.

${ }^{55}$ HAUGH (2017), p. 199.

${ }^{56}$ HAUGH (2017), pp. 200 y s.
} 


\section{Polít. Crim. Vol. 16, № 31 (Junio 2021), Art. 8, pp. 195-216 \\ [http://politcrim.com/wp-content/uploads/2021/05/Vol16N31A8.pdf]}

conducta. ${ }^{57}$ La praxis empresarial muestra que lo único que se consigue con ello es desgastar la organización y a los propios empleados, quienes terminan viendo en el compliance un obstáculo en lugar de un socio del negocio. Aquí las empresas parecen más preocupadas en evitar la sanción que en prevenir los delitos.

Punto de partida para este enfoque del behavioral compliance es detenernos a analizar de qué modo obra este tipo de delincuente. Si bien el conocido Simple Model of Rational Crime (SMORC) de Gary Becker establece que las personas que cometen delitos se basan en un análisis racional de cada situación (es decir, un análisis costo-beneficio), ${ }^{58}$ existen otras motivaciones en juego que hacen que el autor no siempre obre de tal manera. Así, como indica Langevoort, experimentos han demostrado que cuando los sujetos son incitados a mentir, incluso en escenarios sin control ni supervisión, lo hacen rompiendo con la lógica de la maximización de sus beneficios, hasta donde cada uno de ellos es capaz tolerarlo. ${ }^{59}$

La tesis central de Dan Ariely respecto de esta "tolerancia" personal es que nuestra conducta está impulsada por dos motivaciones opuestas. Por un lado, queremos mantener nuestra dosis de imagen positiva (sea cual fuere el nivel que cada uno establezca como adecuado, motivación del ego). Por el otro lado, queremos sacar provecho del engaño y al mismo tiempo seguir considerándonos personas íntegras (motivación económica típica). ${ }^{60}$ De allí surge la máxima que dice que mentimos menos de lo que podríamos, incluso sin riesgo de detección ni sanción, pero más de lo que deberíamos desde un punto de vista ético. ${ }^{61}$

Esto simplemente expone un modo de teorizar acerca de los motivos de comportamientos irregulares dentro de las empresas, sin que con ello tengamos alguna pretensión de ser exhaustivos. Tan solo queremos remarcar la importancia que tiene en el análisis de la adecuación de un sistema de compliance atender a los comportamientos concretos que se dan en el mundo de las organizaciones y estudiar los motivos que los impulsan. Con ello, los líderes de las organizaciones se ven obligados a incorporar al enfoque más formal del compliance un estudio sobre los comportamientos que tienen lugar ad intra. En este punto, las empresas que quieran tener un programa verdaderamente eficaz deberán generar un verdadero cambio cultural, transformador de los valores éticos de quienes trabajan para ellas. ${ }^{62}$

Ahora bien, ¿de qué manera parece más pertinente reaccionar frente a una situación de crisis para generar un cambio cultural en la organización? La primera recomendación pasa por evadir cualquiera de las respuestas más convencionales. Una de las reacciones "en piloto automático" más habituales de las organizaciones ante estos escenarios consiste en sumar más personal, más protocolos y más controles a los programas compliance vigentes, ${ }^{63}$ como si la prevención de delitos en la empresa se redujera a una

\footnotetext{
${ }^{57}$ REA et al. (2016), passim.

${ }^{58}$ Ver ORTIZ DE URBINA GIMENO (2004), pp. 51 y ss.

${ }^{59}$ LANGEVOORT (2012), pp. 949 y s.

${ }^{60}$ ARIELY (2012), p. 25.

${ }^{61}$ BAZERMAN y GINO (2012), p. 85.

${ }^{62}$ MOMSEN y TWEEN (2015), pp. 1043 y s. En este sentido, MOOSMAYER et al. (2018), pp. 58 y ss.

${ }^{63}$ DE CREMER y LEMMICH (2019), passim.
} 
lógica acumulación de políticas, algo que en realidad no agrega nada al cambio de los patrones de comportamiento. ${ }^{64}$

El momento de quiebre en la transformación de la cultura de la concreta organización comienza en realidad con la decisión de abandonar la meta de la mera exoneración de la pena y trabajar en pos de un comportamiento genuinamente ético. Dicho con otras palabras: la meta del compliance ya no sería la exoneración del castigo de la empresa por tener un programa de prevención sólido, sino la exoneración por la inexistencia de ilícitos de personas individuales. Ello exige desechar ciertos hábitos e incorporar otros nuevos. En orden a lo primero, se juzga saludable abandonar o reformular la noción de "lealtad", algo que en el mundo del management resulta fundamental, pues compromete a los miembros de una organización con un fin común. ${ }^{65}$ Sin embargo, puede ser un ingrediente fatal a la hora de tomar consciencia respecto de la peligrosidad de una maniobra concreta, especialmente cuando la finalidad que se persigue es altruista, ${ }^{66}$ no en relación a los propios empleados, sino en relación a la compañía.

Por su parte, entre los nuevos hábitos que se recomienda incorporar aparece la revalorización del diálogo en niveles menos habituales en el management. Más allá de los esfuerzos de criminal compliance, los líderes deben incorporar el diálogo y el intercambio diario con los diversos sectores de la corporación, pero especialmente en la toma de decisiones sobre aspectos relativos a la ética, ${ }^{67}$ de manera de evitar los sesgos propios de la homogenización de pensamiento. ${ }^{68}$ En este sentido, es importante hablar de los ejemplos positivos de la conducta ética esperada por todos los integrantes de la compañía, y no sólo de los casos de error. La conversación multisectorial dentro de la empresa ayuda a delinear el límite de nuestra ética personal y el lugar donde se encuadra la ética corporativa de la organización respectiva.

Por oposición, los sistemas impulsados por el control $^{69}$ crean una cultura en la que aquellos que han transgredido sólo actuarán de manera egoísta para evitar el daño a su propia imagen moral. ${ }^{70}$ En este contexto, toda organización debería perseguir el objetivo de alcanzar una genuina mejora continua, en donde el bien común prime sobre el interés personal, sobre la base del ejemplo que den los líderes con sus acciones. ${ }^{71}$ Crear una organización que fomente una conducta ejemplar puede ser la mejor manera de prevenir una mala conducta perjudicial ulterior. ${ }^{72}$ En este cambio de estrategia suele ser recomendable sumar a los equipos de compliance a profesionales de diversas áreas, tales como psicólogos, sociólogos y especialistas del comportamiento. Ello permitirá incorporar diversidad al proceso de toma de decisiones y darle propósito y sentido a todas y cada una de las acciones de compliance que se emprendan.

\footnotetext{
${ }^{64}$ REA et al. (2016), passim.

${ }^{65}$ LANGEVOORT (2017), p. 934.

${ }^{66}$ ARIELY (2012), pp. 222 y ss.

${ }^{67}$ CARUCCI (2016), passim.

${ }^{68}$ En este punto existen ya experiencias exitosas. Por ejemplo, Bob Kniffin, ex VP de Johnson \& Johnson, asegura que a finales de los 80 ' fueron decisivas para poder manejar de manera eficiente la crisis de Tylenol las llamadas "Credo Challenge Sessions", en las que pequeños grupos de la compañía discutieron acerca de sus perspectivas y compromisos éticos de la firma. Al respecto, confróntese HAUGH (2017), passim.

${ }^{69}$ Crítico respecto a la concepción del compliance guiada por la lógica del control y explicitando sus características, NIETO MARTÍN (2013), pp. 32-ss.

${ }^{70}$ DE CREMER y LEMMICH (2019), passim.

${ }^{71}$ REA et al. (2016), passim.

72 PAINE (1994), passim.
} 
Sin dudas, lo anterior se apoya en un nuevo modo de entender las capacitaciones y la edificación del tono ético de la alta gerencia. En cuanto a lo primero, se torna necesaria la ruptura con el método tradicional de entrenamientos o capacitaciones y la adopción de un cambio en las temáticas y especialmente en los métodos. En este sentido, los entrenamientos deben desarrollarse sobre dinámicas que potencien la interacción con los destinatarios y que de este modo pueda transformarse culturalmente la organización. ${ }^{73} \mathrm{Al}$ mismo tiempo, la alta gerencia debería abandonar la tradición de limitarse a transmitir periódicamente comunicados sobre cuestiones éticas de la corporación o a seguir irreflexivamente un decálogo de buenas prácticas de la alta gerencia. Por el contrario, se requiere una alta gerencia comprometida desde las acciones concretas, especialmente en la adopción de decisiones que, pese a resultar prima facie inconvenientes desde el punto de vista comercial, redundan en la defensa de los valores empresariales.

Aquí tan solo hemos pretendido mostrar algunos lineamientos generales sobre la óptica desde la que resulta necesario trabajar en las organizaciones a nivel de behavioral compliance para lograr tener un sistema verdaderamente eficaz. Se trata, además, de un enfoque que en el diseño e implementación de un sistema de compliance no debería incorporarse al final del proceso, sino que, al contrario, debería impregnar cada una de sus etapas. Sin dudas, estas referencias generales al diálogo y a la integración de equipos interdisciplinarios son sólo un punto de partida.

\section{Reflexiones finales: Replanteamiento de la noción de eficacia}

Claramente un modelo de tres filtros eleva notablemente los estándares de valoración sobre la adecuación de un sistema de compliance, especialmente si consideramos la exigencia que se presenta en el último paso. En general, se aprecia tanto en la discusión académica como en los lineamientos establecidos por organismos públicos de diferentes países que los estándares mínimos de calidad están vinculados con la satisfacción de los requisitos de los primeros dos filtros. Como se enfatizó al momento de caracterizar el denominado behavioral compliance, este tercer nivel de exigencias fue recomendado sólo en tiempos recientes sobre la base de profundizar en las razones que explican los comportamientos desviados en las empresas. Ahora bien, la significativa elevación de los estándares que supone el modelo de los tres filtros nos obliga a formular una pregunta decisiva para la praxis de las personas jurídicas: ¿Puede exigirse en la praxis a las personas jurídicas que sus modelos de compliance pasen los tres filtros?

Indudablemente la respuesta dependerá de cada país y específicamente del nivel de desarrollo que tenga allí la cultura del compliance. La necesidad de satisfacer estos estándares no parece disparatada en EE.UU. o el Reino Unido, donde existe una profunda cultura de cumplimiento, instalada en las organizaciones desde hace ya varias décadas. Por el contrario, incluso en Europa, donde desde comienzos del siglo XXI el fenómeno autorregulatorio ha experimentado un notable crecimiento en la praxis y en la ciencia, la exigencia de superar un tercer filtro resulta exagerado. Más aún lo es en un contexto como el latinoamericano donde se trata todavía de un fenómeno incipiente, con un soporte institucional bastante endeble y con una praxis que oscila entre el desconocimiento y el escepticismo respecto a la lucha contra la corrupción. No sería extraño pensar que en

73 Sobre las características que debería asumir el nuevo formato de entrenamientos, confróntese KLEINHEMPEL (2018), pp. 219 y ss. 
estos contextos una sobre exigencia podría ser contraproducente. De hecho, consideramos que bajo este razonamiento operó el legislador argentino cuando en el art. 23 Ley $\mathrm{N}^{\circ}$ 27.401 estableció estándares mínimos demasiado bajos, buscando un alto acatamiento por parte del empresariado de las prácticas de compliance, olvidando que así únicamente se estaba incentivando la proliferación de un make-up-compliance. Esto nos lleva a tener que distinguir en esta etapa de evolución entre la adecuación y la eficacia de un programa de compliance, términos que hasta ahora aparecen vinculados como si fuesen sinónimos. ${ }^{74}$

Más bien, consideramos que sería conveniente desvincular ambas nociones. Entendemos que un sistema es adecuado en la medida en que goza de una solidez tal que solamente mediante sofisticadas maniobras de los delincuentes podría verse doblegado para impedir la comisión de un delito. Ello significa que la adecuación está sujeta a que el sistema cuente con los elementos requeridos por el tipo de riesgo ligado a la actividad, su dimensión y su capacidad económica y que ellos funcionen de tal manera que únicamente resulte vulnerable ante enormes esfuerzos defraudatorios. Esto puede lograrse incluso en personas jurídicas que no están convencidas del valor de la integridad y que únicamente se autorregulen para cumplir con la ley o por puras razones comerciales. Para ello, no se requiere necesariamente un total cambio cultural en la organización que modifique las razones subyacentes a la adopción de un sistema de compliance. Hasta aquí, las prácticas regulatorias a nivel comparado han exigido un programa adecuado para exonerar a las personas jurídicas de responsabilidad penal. ${ }^{75}$ Bastaría entonces para eludir la sanción cumplir con los primeros dos filtros, pero ello todavía no podría dejar totalmente tranquila a la organización, pues podría existir un "caldo de cultivo de ilicitudes" residual que favorezca la aparición de comportamientos desviados.

La eficacia del programa justamente tiene que ver con esto último, esto es, representa un plus en relación a la adecuación. Un sistema funcionalmente adecuado sirve para satisfacer lo estándares de compliance reconocidos por países experimentados en estas materias, pero no deberían generar una absoluta tranquilidad en los empresarios hasta tanto no se produzca un genuino cambio cultural favorable al compliance. Con un sistema de compliance que cumple con los tres filtros la empresa no solamente estará en condiciones de evitar multas de las entidades reguladoras, sino también (y fundamentalmente) de verse involucrada en hechos de corrupción. La eficacia en un programa de compliance marca un salto cualitativo desde el estadio de buscar evadir sanciones a la prevención efectiva de delitos. Creemos que una organización que no ha internalizado profundamente la cultura ética y del cumplimiento en sus negocios nunca podrá eliminar completamente los riesgos de verse involucrada en ilicitudes. Un programa de compliance adecuado reduce drásticamente los riesgos de que se comentan delitos, pero siempre deja abierto un margen (más o menos pequeño) de riesgos derivados de la inexistencia de un compromiso genuino de la alta gerencia y de los restantes miembros de la corporación. Por tanto, solamente una organización que haya trabajado desde la perspectiva del behavioral compliance podrá afirmar que goza de un programa de compliance adecuado y eficaz.

\footnotetext{
74 Así, por ejemplo, en WELLNER (2005), pp. 500 y ss.; MUÑOZ DE MORALES ROMERO (2013), pp. 211 y ss.

${ }^{75}$ La noción de adecuación podría significar aquí que el sistema de compliance tenga la capacidad de impedir seriamente la comisión de delitos. Así, confróntese BOCK (2011), p. 461.
} 
Justamente por las razones expuestas anteriormente no resulta conveniente que la exención de responsabilidad de la persona jurídica quede sujeta a la eficacia del sistema, sino que para ello basta con su adecuación. Un sistema de compliance adecuado satisface en este estadio del desarrollo las exigencias mínimas que deben darse para afirmar que una persona jurídica no padece de defectos de organización. Todavía hoy la eficacia en los términos planteados aquí aparece como una pretensión supererogatoria para las empresas, pero que — nos animamos a augurar — en las próximas décadas se transformará en una exigencia natural para organizaciones que quieren evitar una responsabilidad (penal o administrativa) por la comisión de delitos. 


\section{Bibliografía citada}

AGUSTINA, José; VARGAS OVALLE, Alejandra (2019): "Informe al borrador de los «Lineamientos»", en: MONTIEL, Juan Pablo (Dir.), Lineamientos de integridad (Buenos Aires, CRIMINT) pp. 87-91.

ARIELY, Dan (2012): The (Honest) Truth about Dishonesty (Nueva York, Harper Perennial).

BAZERMAN, Max; GINO, Francesca (2012): "Behavioral Ethics: Toward a Deeper Understanding of Moral Judgement and Dishonesty", en: Annual Review of Law and Social Science ( $\left.{ }^{\circ} 8\right)$, pp. 85-104.

BERMEJO, Mateo (2013): "Delincuencia empresarial: la regulación del informante interno (whistleblower) como estrategia político criminal”, en: Revista Derecho penal ( $\left.\mathrm{N}^{\mathrm{o}} 4\right)$, pp. 52-75.

BERNDT, Thomas; JABLOWSKI, Lea-Victoria (2019): "Berichterstattung zur Bekämpfung von Korruption und Bestechung in der nicht-finanziellen Erklärung -eine Analyse der DAX-30-Unternehmen”, en: Compliance Berater, pp. 110-117.

BOCK, Dennis (2011): Criminal Compliance (Baden Baden, Nomos).

CARUCCI, Ron (2016): "Why Ethical People Make Unethical Choices", en: Harvard Business Review (December). Disponible en: https://tinyurl.com/ygoambwp [visitado el 12/12/2019].

CHEN, Hui; SOLTES, Eugene (2018): "Why Compliance Programs Fail - and How to Fix Them", en: Harvard Business Review ( $\mathrm{N}^{\circ}$ Marzo-Abril). Disponible en: https://hbr.org/2018/03/why-compliance-programs-fail [visitado el 27/11/2019].

CHEREPANOVA, Vera (2019): "ISO 37.001: Not All Certifications are Created Equal", en: The FCPA Blog. Disponible en: https://tinyurl.com/yfn8eaeo [visitado el 03/04/2019].

CORIA CARO, Carlos; SOTA SÁNCHEZ, André (2019): "Reporte al Borrador de los «lineamientos»", en: MONTIEL, Juan Pablo (Dir.), Lineamientos de Integridad (Buenos Aires, CRIMINT), pp. 103-113.

DAWSON, Ian; DUNN, Alison (2006): "Governance Code of Practice in the Non-For Profit Sector", en: Corporate Governance (Vol. 14, No 1), pp. 33-42.

DE CREMER, David; LEMMICH, Bjarne (2019): "Compliance Alone Won't Make Your Company Safe", en: Harvard Business Review (Mayo). Disponible en: https://tinyurl.com/yz2pwhsz [visitado el 12/12/2019].

GARCÍA CAVERO, Percy (2017), Criminal Compliance, (Lima, Ideas).

GÓMEZ-JARA DIÉZ, Carlos (2010), “¿Responsabilidad penal de todas las personas jurídicas? Una antecrítica al símil de la ameba acuñado por Alex Van Weezel”, en: Política Criminal ( ${ }^{\circ}$ 5), pp. 455-475.

GÓMEZ-JARA DIÉZ, Carlos (2016): “Tomarse la responsabilidad penal de las personas jurídicas en serio: la culpabilidad de las personas jurídicas”, en: Revista En Letra Derecho Penal (2016, N²), pp. 24-39.

GÓMEZ MARTÍN, Víctor (2013): "Compliance y Derecho de los trabajadores", en: KUHLEN, Lothar; MONTIEL, Juan Pablo; ORTIZ DE URBINA, Iñigo (eds.), Compliance y Teoría del Derecho penal (Madrid, Barcelona, Buenos Aires, Sao Paulo, Marcial Pons), pp. 125-146.

HAUGH, Todd (2017): “Cadillac Compliance Breakdown”, en: Stanford Law Review Online (April). Disponible en: https://tinyurl.com/yg7r7tqs [Visitado el 12/12/2019].

HEMPHILL, Thomas; CULLARI, Francine (2009), "Corporate Governance Practices: A Proposed Policy Incentive Regime to Facilitate Internal Investigations and Self 
Reporting of Criminal Activities", en: Jounal of Business Ethics ( $\mathrm{N}^{\circ}$ 87), pp. 333 351.

HERNÁNDEZ BASUALTO, Héctor (2017): "La introducción de la responsabilidad penal de las personas jurídicas en Chile”, en: Política Criminal (vol. 5), pp. 207 236.

HILF, Johanna (2015), "§ 9 Die strafrechtliche Verantwortlichkeit juristischer Person im Rechtsvergleich unf ihre Auswirkungen auf Criminal Compliance: Schweiz, Österreich, Liechtenstein", en: ROTSCH, Thomas (Ed.), Criminal Compliance (Baden Baden, Nomos), pp. 289-312.

KLEINHEMPEL, Matthias (2018): “Comunicación, formación y concientización: ¿cómo hacer un programa de integridad efectivo", en: SACCANI, Raúl; DURRIEU, Nicolás (Comp.), Compliance, Anticorrupción y responsabilidad penal empresaria, (Buenos Aires, La Ley), pp. 219-227.

KNIERIM, Thomas (2015), "§ 6 B Aus Sicht des externen Compliance-Beraters", en: ROTSCH, Thomas (Ed.), Criminal Compliance, (Baden Baden, Nomos), pp. 212 224.

KUBICIEL, Michael (2015): “Compliance als Strafausschlussgrund in einem künftigen Unternehmensstrafrecht", en: AHLBRECHT, Heiko; DANN,Matthias;

WESSING, Helga; FRISTER, Helmut; BOCK, Dennis (Comp.), Unternehmensstrafrecht. Festschrift für Jürgen Wessing (Múnich, C. H. Beck), pp. 69-83.

LANGEVOORT, Donald (2017): "Cultures of Compliance", en: American Criminal Law Review (2017, Vol. 54), pp. 933-977. Disponible en: https://tinyurl.com/yzto6lvb [Visitado el 12/12/2019].

LEHMANN, Erik (2015): "§ 3 Unternehmensorganisation und Criminal Compliance”, en: ROTSCH, Thomas (Ed.), Criminal Compliance (Baden Baden, Nomos), pp. 101141.

MARTÍNEZ, Diego (2018): “La evaluación de los programas de integridad. La posible interpretación y aplicación de los arts. 22 y 23 de la ley 27.401 en el ámbito del proceso penal”, en: SACCANI, Raúl; DURRIEU, Nicolás (Comp.), Compliance, Anticorrupción y responsabilidad penal empresaria (Buenos Aires, La Ley), pp. 287-301.

MOLANDER, Earl (1987): “A paradigm for design, promulgation and enforcement of ethical codes", en: Journal of Business Ethics (No 6), pp. 619-631.

MOMSEN, Claus; TWEEN, Douglas (2015): "§ 30 Criminal Compliance in den USA, der EU und Großbritannien”, en: ROTSCH, Thomas (Ed.), Criminal Compliance (Baden Baden, Nomos), pp. 1027-1057.

MOMSEN, Claus (2015): “Interne Ermittlungen", en: ROTSCH, Thomas (Ed.), Criminal Compliance (Baden Baden / Nomos), pp. 1234-1260.

MONTANER FERNÁNDEZ, Raquel (2019): "Informe de valoración sobre el contenido del borrador de los Lineamientos", en: MONTIEL, Juan Pablo (Dir.), Lineamientos de Integridad (Buenos Aires, CRIMINT), pp. 93-99.

MONTANER FERNÁNDEZ, Raquel; FORTUNY, Miquel (2018): “La exención de responsabilidad penal de las personas jurídicas: Regulación jurídico-penal vs. UNE 19601", en: La Ley Penal (Volumen 132, Número mayo-junio), pp 1-19.

MONTIEL, Juan Pablo (2018a): "Lineamientos de integridad: breves reflexiones generales del documento y consideraciones específicas sobre las capacitaciones, las investigaciones internas y el oficial de cumplimiento", en: MONTIEL, Juan Pablo (Dir.), "Lineamientos de Integridad". Disponible en: https://tinyurl.com/yz8a9nkk [visitado el 14/05/2021], pp. 11-18. 
MONTIEL, Juan Pablo (2018b): "Sobre la inoponibilidad de la prescripción y de la cosa juzgada en casos de corrupción (pública)”, en: GÓMEZ MARTÍN, Víctor; MONTIEL, Juan Pablo; SATZGER, Helmut (Eds.), Estrategias penales y procesales de lucha contra la corrupción (Madrid, Marcial Pons), pp. 171-198.

MONTIEL, Juan Pablo (2018c): "Ley 27.401 y criterios para determinar la idoneidad de los programas de integridad", en: SACCANI, Raúl; DURRIEU, Nicolás (Comp.), Compliance, Anticorrupción y Responsabilidad Penal Empresaria (Buenos Aires, La Ley), pp. 75-83.

MONTIEL, Juan Pablo (2018d), "Apuntes sobre el nuevo régimen de responsabilidad penal de las personas jurídicas en el Derecho Argentino", en: Revista En Letra Derecho Penal (Volumen, Número 6), pp. 124-149.

MONTIEL, Juan Pablo (2012): "Unternehmerische Selbstreinigung: Compliance Programme, interne Untersuchungen und Neutralisierung strafrechtlicher Risiken", en: KUHLEN, Lothar; KUDLICH, Hans; ORTIZ DE URBINA, Iñigo (Eds.), Compliance und Strafrecht (Heidelberg; Múnich; Landsberg; Frechen; Hamburgo, C. F. Müller), pp. 185-208.

MOOSMAYER, Klaus; KRONEN, Daniel; VON REUTERN-KULENKAMP, Corinna (2018): "El día después: ¿Cómo fortalecer la cultura de Compliance e integridad después de una sanción”, en: SACCANI, Raúl; MORALES OLIVER, Gustavo (Dir.), Tratado de Compliance, tomo II, (Buenos Aires, La Ley), pp. 58-73.

MUÑOZ DE MORALES ROMERO, Marta (2013): "Programas de cumplimiento 'efectivos' en la experiencia comparada", en ARROYO ZAPATERO, Luis; NIETO MARTÍN, Adán (Dir.), El Derecho penal económico en la era del compliance (Valencia, Tirant lo Blanch), pp. 211-230.

NIETO MARTÍN, Adán (2013): "Problemas fundamentales del cumplimiento normativo en el Derecho penal", en: KUHLEN, Lothar; MONTIEL, Juan Pablo; ORTIZ DE URBINA, Iñigo (Eds.), Compliance y Teoría del Derecho penal, (Buenos Aires, Marcial Pons), pp. 32-46.

NIETO MARTÍN, Adán (2015): "Cumplimiento normativo, criminología y responsabilidad penal de las personas jurídicas”, en: NIETO MARTÍN, Adán (Dir), Manual de Cumplimiento Penal en la Empresa (Valencia, Tirant lo Blanch), pp. 80-108.

ORTIZ DE URBINA GIMENO, Iñigo (2004): “Análisis económico del Derecho y política criminal”, en: Revista de Derecho penal y Criminología ( $\left.\mathrm{N}^{\circ} 2\right)$, pp. 31-74.

PALMA HERRERA, José Manuel (2014): "El papel de lo compliance en un modelo vicarial de la responsabilidad penal de la persona jurídica", en: GONZÁLEZ TAPIA, María Isabel; PALMA HERRERA, José Manuel (Dir.), en: Procedimientos operativos estandarizados y responsabilidad penal de la persona jurídica (Madrid, Dykinson), pp. 157-231.

REA, Peter; KOLP, Alan; RITZ, Wendy; STEWARD, Michelle (2016): "Corporate Ethics Can't Be Reduced to Compliance", en: Harvard Business Review (April). Disponible en: https://tinyurl.com/yfp3ltsj [visitado el 12/12/2019].

REEB, Philipp (2011): Internal Investigations, (Berlín, Duncker \& Humblot).

ROTSCH, Thomas (2015): "Grundlagen der Criminal Compliance", en: ROTSCH, Thomas (Ed.), Criminal Compliance (Baden Baden, Nomos), pp. 82-100.

SCHEFOLD, Christian (2017), ISO 37001 Compliance. Anforderungskatalog und Guidance für das Unternehmens-CMS zur Bestehungspreväntion, en: ZFRC (No 1), pp. 27-34.

STEVENS, Betsy (2008): "Corporate Ethical Codes: Effective Instruments For Influencing Behavior”, en: Jounal of Business Ethics (N 78), pp. 601-609. 
WAUSCHKUHN, Antonie (2012): "Sanktionierung nach Abschluss der Untersuchungen”, en MOOSMAYER, Klaus; HARTWIG, Niels (Eds.), Interne Untersuchungen (Múnich, C.H. Beck), pp. 67-80.

WELLNER, Philip (2005): "Effective compliance programs and corporate criminal persecutions", en: Cardozo Law Review (No 27:1), pp. 497-528.

ZENTES, Christina (2017): Das Sieben-Säulen-Modell zur Korruptionsprävention (Frankfurt Main/ Springer).

\section{Otros documentos citados}

ISO N 37001

FCPA (2018): "Enforcement Report 2018, 2017, 2016", en: The United States Department of Justice. Disponible en: https://tinyurl.com/yz9a7jtg [Visitado el 10/07/2019].

FCPA Enforcement Report 2018, 1017 \& 2016, Obtenido el 10 de julio de 2019

Guía Práctica. Buenas Prácticas de Investigación. Responsabilidad penal de las personas jurídicas (Chile).

Lineamientos de Integridad para el mejor cumplimiento de lo establecido en los artículos 22 y 23 de la ley $\mathrm{n}^{\circ} 27.401$ de responsabilidad penal de las personas jurídicas (Argentina).

U.S. Department of Justice, Evaluation of Corporate Compliance Programs (Estados Unidos de América).

U.S. Sentencing Guidelines (Estados Unidos de América). 